\title{
Diseño de un proceso para la obtención de una bebida nutritiva a base de Hortalizas como el tomate (Solanum lycopersicum) y el pepinillo (Cucumis sativus)
}

Design of a process for the obtaining Of a nutritive drink based on vegetables like tomato (solanum lycopersicum) and pepinillo (cucumis sativus)

Sonia Mercedes Vallejo Abarca . ${ }^{1}, \&$ Edmundo Caluña Sánchez. ${ }^{2}$

Recibido: 10-04-2019 / Revisado: 15-05-2019 /Aceptado: 24-06-2019/ Publicado: 05-07-2019

\begin{abstract}
.
DOI: https://doi.org/10.33262/cienciadigital.v3i3.662

The present research proposes the use of vegetables, bulbs and tuber to prepare a nourishing and hydrating drink as a dietary supplement for a healthy lifestyle, in which different levels of tomato $(28 \%, 38 \%$ and $48 \%)$ were added and pickle $(20 \%, 30 \%$ and $40 \%$ ) to a mixture of peppers, onions, garlic and carrots also adding ingredients that enhanced the taste of the drink.

Once the drinks obtained underwent physical tests - chemical and microbiological tests, in order to determine its nutritional quality and were sensory evaluated to establish its acceptability with the result that the drink made with cucumber and different levels of tomato gained wider acceptance when $48 \%$ of tomato was used whose variables accounted for 10 points recorded the highest values regarding levels tomato 28 and $38 \%$, because the tomato provides better sensory characteristics of color, odor, taste and appearance, to thereby accept the alternative hypothesis raised in the investigation.
\end{abstract}

Keywords: Vegetables, Bulbs, Tubers, Tomatoes, Cucumber, Drink, Acceptability.

\footnotetext{
1 Escuela Superior Politécnica de Chimborazo, Facultad de Ciencias. Riobamba, Ecuador. sonia.vallejo@espoch.edu.ec

2 Escuela Superior Politécnica de Chimborazo, Facultad de Ciencias. Riobamba, Ecuador. ecaluna2010@ hotmail.com
} 


\section{Resumen.}

Se programa realizar una bebida nutritiva utilizando hortalizas, bulbos y tubérculos como complemento alimenticio para una vida saludable, en la que se incorporaron diferentes niveles de tomate $(28 \%, 38 \%$ y $48 \%)$ y pepinillo $(20 \%, 30 \%$ y $40 \%)$ a un coctel de cebollas, pimientos, zanahorias y ajo al que se le adicionó otros ingredientes que realzaron los atributos sensoriales a la bebida.

A las bebidas obtenidas se les realizó pruebas físico - químicas, microbiológicas y sensoriales con el objeto de determinar su valor nutritivo y aceptabilidad resultando que la bebida elaborada con pepinillo y $48 \%$ de tomate fue la mayormente aceptada con respecto a los niveles 28 y $38 \%$ de tomate, la calificación de las variables fue sobre 10 puntos registrándose los valores más altos debido a que el tomate le proporciona mejores características sensoriales a la bebida.

Palabras claves: Hortalizas, Bulbos, Tubérculos, Tomate, Pepinillo, Bebida, Aceptabilidad.

\section{Introducción.}

Un estilo de vida saludable es consecuencia directa del consumo de alimentos nutritivos y bajos en grasas como son las hortalizas frescas que conforman un grupo heterogéneo alimenticio de las que pueden ser aprovechadas y consumidas desde sus frutos, flores, bulbos, tubérculos, tallos, brotes y hojas que constituyen un todo a nivel de la planta.

Razón por la que hay que recalcar que el consumo se hortalizas identifica un estilo de vida saludable y equilibrado corroborado por grupos de personas que han experimentado cambios positivos al incorporar este tipo de productos en su dieta diaria, convirtiéndose de esta manera en un grupo de alimentos básicos para la alimentación humana.

El término hortaliza se refiere a todas las plantas herbáceas que se cultivan generalmente en huertos y son adecuadas para el consumo humano, ya sean crudas o cocinadas. Por su gran contenido de agua estos alimentos contribuyen a hidratar nuestro organismo, además de ser nutritivas y saludables.

El consumo de hortalizas frescas se ha convertido en una actitud que identifica un estilo de vida saludable y equilibrado por parte de grupos de personas que han experimentado cambios positivos al incorporar este tipo de productos en su dieta diaria, en consecuencia, parece claro que se trata de un conjunto de productos básicos dentro de la alimentación humana. En este sentido, hay que recalcar que las hortalizas constituyen un grupo heterogéneo que incluye un gran número de especies utilizadas por el hombre; pueden aprovecharse de las hortalizas las raíces, los frutos, las flores, los bulbos, los tubérculos, los tallos, los brotes y las hojas que constituyen un todo a nivel de la planta. 
Los zumos y bebidas a base de frutas son comúnmente encontrados en el mercado y tienen la finalidad de ser incorporados a la dieta de las personas para contribuir al cambio de su estilo de vida lográndose demostrar que su consumo contribuye a reducir el riesgo de enfermedades de alto impacto, entre ellas las cardiovasculares, degenerativas, infantiles, obesidad mórbida y el cáncer como consecuencia del consumo de comidas que no aportan con ningún nutriente en la dieta de una persona lo que conlleva al deterioro de su salud.

Por lo expuesto anteriormente se quiere incorporar a la dieta humana una bebida a base de hortalizas, bulbos y tubérculos de los que se aprovechan sus nutrientes y atributos sensoriales para mejorar el estilo de vida de las personas que lo consuman.

\section{Metodologia.}

\section{Localización y duración del experimento}

El trabajo investigativo se llevó a cabo en el Laboratorio de Procesos Industriales de la Facultad de Ciencias de la ESPOCH, ubicada en el Kilómetro 1 1 1/2 de la Panamericana Sur, a una altitud de 2740 m.s.n.m con una latitud de 01 o 32 ' S y una longitud de 78 o 40 ' W.

\section{Unidades experimentales}

Por el efecto de los niveles de pepinillo (20,30 y $40 \%)$ y niveles de tomate $(28,38$ y $48 \%)$ en las que se realizaron 3 repeticiones, se manejaron 27 unidades experimentales, siendo el TUE (tamaño de la unidad experimental) de un (1) litro de la bebida.

\section{Tratamiento y diseño experimental}

Los tratamientos se definieron en base a dos factores:

Factor A con Tomate (28, 38 y 48\%)

Factor B con Pepinillo (20, 30 y 40\%).

Las unidades experimentales fueron modelados bajo un Diseño Completamente al Azar (DCA) con arreglo combinatorio. 


\section{Análisis estadístico y pruebas de significancia}

Los resultados de la investigación tabulados y procesados se sometieron a los análisis estadísticos que señalamos a continuación:

Análisis de varianza (ADEVA) para las diferencias entre tratamientos.

Las comparaciones múltiples se realizaron mediante la prueba de Duncan a los niveles de significancia $\mathrm{p}<0.05$.

\section{Procedimiento Experimental}

Se formuló la bebida, adicionando diferentes niveles de pepinillo (20,30 y 40\%) y tomate $(28,38$ y $48 \%)$ a una mezcla de pimientos $(20 \%)$, cebollas (10\%), ajos (1\%) y zanahoria (1\%); además se incluyeron otros ingredientes que realzaron el sabor de la bebida (aceite, vinagre, agua y sal), a continuación, se describe el procedimiento para la obtención de la bebida.

1. Selección de la materia prima (tomate, pepino, pimiento, cebolla, zanahoria y ajo).

2. Una vez seleccionada la materia prima se realiza una limpieza y desinfección de la misma con el objeto de eliminar la carga microbiana superficial.

3. Realizamos un acondicionamiento de las hortalizas mediante cortado y pelado y que se lo hará de forma manual.

4. Posteriormente procedemos a pesar y estandarizar las materias primas e ingredientes.

5. Una vez que las materias primas están pesadas las mezclamos y acidificamos con el empleo de ácido ascórbico.

6. Luego adicionamos aceite de oliva y mezclamos.

7. Dejamos que las hortalizas se maceren durante 24 horas y en refrigeración.

8. Al macerado adicionamos agua, sal y el resto de ingredientes y procesamos.

9. Posteriormente envasamos en botellas de vidrio.

10. Esterilizamos el producto en una olla autoclave a $115 \mathrm{oC}$ durante $15-20$ minutos

11. Finalmente enfriamos el producto para su posterior almacenamiento. 
Gráfico No.1. Procedimiento experimental

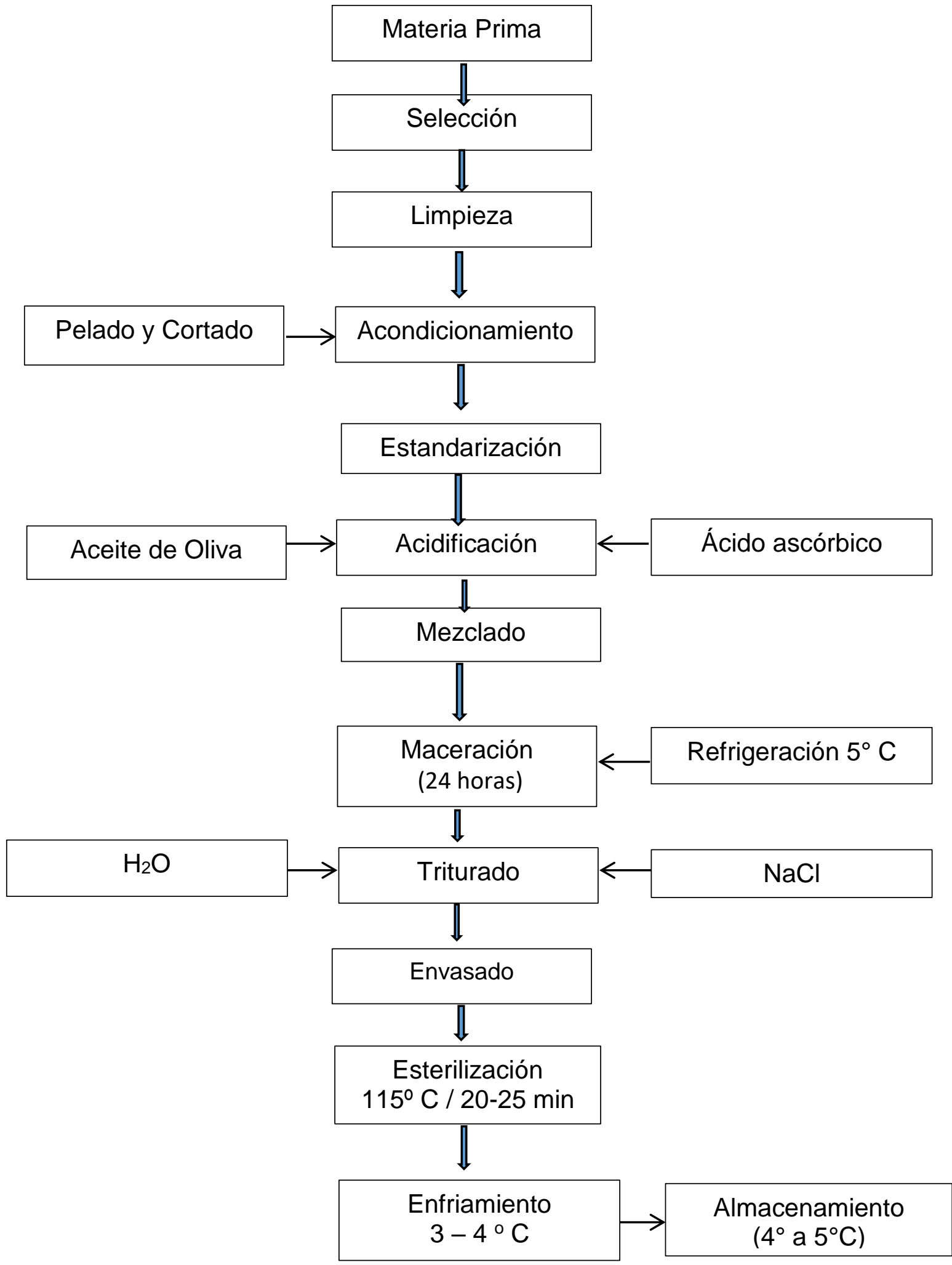

Fuente: Vallejo, S. (2014) 


\section{Resultados.}

\section{Análisis Bromatológicos}

Los resultados obtenidos de los análisis bromatológicos que se realizaron a las bebidas se reportan en las Tablas de datos, por lo que se analizaron de acuerdo a cada variable físico química en estudio y se detalla a continuación:

\section{Humedad}

El contenido de humedad de la bebida elaborada con pepinillo y diferentes niveles de tomate (28\%, 38\% y 48\%), de acuerdo al ADEVA, se encontraron diferencias altamente significativas $(\mathrm{P}<0.01)$, por efecto de los niveles de tomate empleados, determinándose el mayor contenido de humedad 81,12\% cuando se empleó $48 \%$ de tomate, sin embargo se observa también que existe un menor contenido de humedad 80,62 \% cuando se utilizó el $28 \%$ de tomate, mientras que al considerar el factor pepinillo también se establecieron diferencias altamente significativas $(\mathrm{P}<0.01)$, por efecto de los niveles empleados $(20 \%, 30 \%$ y $40 \%$ ), encontrándose el mayor contenido de humedad $80,95 \%$ cuando se empleó $40 \%$ y el menor contenido $80,78 \%$ al utilizarse en la formulación de la bebida $20 \%$ de pepinillo.

En otros estudios realizados del contenido de agua en bebidas alimenticias elaboradas a base de lactosuero con la inclusión de tres niveles de pulpa de pitahaya, Yumisaca, C. (2009) reporta que fluctúa entre $74,54 \%$ y $72,68 \%$ resultados que comparados con los obtenidos en la presente investigación son inferiores ya que las hortalizas bulbos y tubérculos empleados como materia prima para la bebida tienen gran contenido de agua a diferencia de la fruta utilizada en la investigación citada.

\section{Lípidos}

Las medias del contenido de grasa de la bebida elaborada con pepinillo y diferentes niveles de tomate $(28 \%, 38 \%$ y $48 \%)$ presentaron diferencias altamente significativas $(\mathrm{P}<0.01)$, encontrándose el mayor contenido de grasa $15,55 \%$ cuando se empleó $48 \%$ de tomate, sin embargo se observa también que existe un menor contenido de grasa 15,01\% cuando se utilizó en la formulación de la bebida el $28 \%$ de tomate, se encontraron también diferencias altamente significativas $(\mathrm{P}<0.01)$, por efecto de los niveles de pepinillo $(20 \%, 30 \%$ y $40 \%)$ empleados reportándose el mayor contenido de grasa de $15,37 \%$ cuando se empleó $40 \%$ de pepinillo y un menor contenido $15,19 \%$ cuando se utilizó en la formulación de la bebida el $20 \%$ de pepinillo.

El contenido de grasa en las sopas y bebidas a base de vegetales, según (Fálder Rivero 2004), señala que se encuentra en un porcentaje mínimo del 0,4\% que a diferencia de los resultados obtenidos en la bebida a base de hortalizas bulbos y tubérculos se consiguen valores muy 
elevados que van desde $15,55 \%$ hasta $15,01 \%$, debido a que en la formulación de bebida se empleó como ingrediente aceite de oliva extra virgen en cantidades considerables, mismo que realzó las propiedades organolépticas de la bebida e influyo en el elevado contenido de lípidos reportado, sin dejar de considerarse por esta variable una bebida dietética ya que según (Fálder Rivero 2004) las grasas de este aceite es grasa vegetal con el 99\% de ácidos grasos monoinsaturados y el $1 \%$ restante formado por vitamina $\mathrm{E}$ y antioxidantes naturales necesarios para nuestro organismo.

\section{Proteínas}

El contenido de proteína de la bebida elaborada con pepinillo y diferentes niveles de tomate $(28 \%, 38 \%$ y $48 \%)$, se reportaron diferencias altamente significativas $(\mathrm{P}<0.01)$, por efecto de los niveles de tomate utilizados, encontrándose el mayor contenido de proteína 3,10\% cuando se empleó $48 \%$ de tomate, sin embargo se observa también que existe un menor contenido de proteína 2,02 \% cuando se utilizó en la formulación de la bebida el $28 \%$ de tomate, mientras que en la formulación de la bebida elaborada con tomate y diferentes niveles de pepinillo $(20 \%, 30 \%$ y $40 \%)$, se encontraron diferencias altamente significativas $(\mathrm{P}<0.01)$, por efecto de los mismos, encontrándose el mayor contenido de proteína 2,74\% cuando se empleó $40 \%$ y el menor contenido $2,38 \%$ cuando se utilizó el $20 \%$ de pepinillo.

Según (Cedeño 2005), manifiesta que el valor de proteína en las bebidas nutricionales a base de frutas y hortalizas con leche debe estar entre $3,0 \%$ y 3,45, valores que medidos con los obtenidos en la bebida a base de hortalizas, bulbos y tubérculos, son superiores debido a la presencia de un producto lácteo que incrementa el porcentaje de proteína en la bebida, de la misma manera Crocco Alicia (2014), publica que la sopa de vegetales tiene hasta el $2 \%$ de proteínas que comparados con los resultados obtenidos en la presente investigación se muestran valores que van desde 2,02\% y 3,10\% siendo superiores debido a la combinación de nutrientes tomados de las materias primas del estudio.

\section{Cenizas}

De acuerdo al Análisis de varianza que reporta los resultados del contenido de cenizas de la bebida en estudio elaborada con pepinillo y diferentes niveles de tomate (28\%, 38\% y $48 \%)$, se encuentran diferencias altamente significativas $(\mathrm{P}<0.01)$, por efecto de los niveles de tomate empleados, encontrándose el mayor contenido de cenizas 4,03\% cuando se empleó $48 \%$ de tomate, sin embargo se observa también que existe un menor contenido de cenizas $3,13 \%$ cuando se utilizó en la formulación de la bebida el 28\% de tomate, mientras que al considerar el factor pepinillo $(20 \%, 30 \%$ y $40 \%)$ en la formulación de la bebida con tomate se encontraron diferencias altamente significativas $(\mathrm{P}<0.01)$, por efecto de los niveles de pepinillo, encontrándose el mayor contenido de cenizas 3,73\% cuando se empleó $40 \%$ de 
pepinillo y un menor contenido 3,43\% cuando se utilizó en la formulación de la bebida el $20 \%$ de pepinillo.

Tomando como referencia los estudios de Limones, K y García, M (2011), quienes al elaborar una sopa instantánea a partir de harina de chocho determinaron un contenido de cenizas en el producto de $9,11 \%$ que es un valor superior al de ésta investigación pudiendo deberse a que se consiguieron mayor cantidad de sólidos que los que se obtuvieron en ésta investigación.

\section{pH}

Con respecto al $\mathrm{pH}$ de la bebida elaborada con pepinillo y diferentes niveles de tomate (28\%, $38 \%$ y 48\%), según los análisis estadísticos y de acuerdo al ADEVA se reportaron diferencias altamente significativas $(\mathrm{P}<0.01)$, por efecto de los niveles de tomate, encontrándose un $\mathrm{pH}$ de 4,61cuando se empleó $48 \%$ de tomate, sin embargo se observa también que existe un menor $\mathrm{pH}$ 4,35 cuando se utilizó en la formulación de la bebida el $28 \%$ de tomate, en la formulación de la bebida con tomate y diferentes niveles de pepinillo ( $20 \%$, $30 \%$ y 40\%), no se encontraron diferencias estadísticas $(\mathrm{P}<0.05)$, por efecto de los niveles de pepinillo empleados reportándose un mayor $\mathrm{pH} 4,56$ cuando se empleó $40 \%$ de pepinillo y un menor $\mathrm{pH} 4,449$ cuando se utilizó en la formulación de la bebida el $20 \%$.

El pH es un factor muy importante que se tomó en consideración como punto de control en el proceso, que confrontando con los estudios realizados por Yumisaca, C. (2011), quién al elaborar bebidas nutritivas a partir de lactosuero y concentrados de frutas reportó valores entre 4,7 y 6,5 que comparados con los que se obtuvieron en esta investigación se encuentran dentro de este rango debido a que ambas bebidas son ligeramente ácidas por la adición de lactosuero y de vinagre en la formulación de la bebida a base de hortalizas, bulbos y tubérculos que es una de las razones por la que el producto se conserve por mayor tiempo.

\section{Fibra}

De acuerdo a los análisis estadísticos realizados en lo concerniente al contenido de fibra de la bebida elaborada con pepinillo y diferentes niveles de tomate $(28 \%, 38 \%$ y $48 \%)$ existen diferencias altamente significativas $(\mathrm{P}<0.01)$, por efecto de los niveles de tomate empleado con un mayor contenido de fibra $1,76 \%$ cuando se empleó $48 \%$ de tomate, y un menor contenido de fibra 1,29 \% cuando se utilizó en la formulación el $28 \%$ de tomate, mientras que al considerar el factor pepinillo en la formulación con tomate y diferentes niveles de pepinillo $(20 \%, 30 \%$ y $40 \%)$, se encontraron diferencias altamente significativas $(\mathrm{P}<0.01)$, por efecto de los niveles de pepinillo, reportándose el mayor contenido de fibra $1,6 \%$ cuando se empleó $40 \%$ de pepinillo y un menor contenido 1,443\% cuando se utilizó en la formulación de la bebida el $20 \%$ de pepinillo. 
El contenido de fibra presente en las sopas y caldos de hortalizas y vegetales según (Fálder Rivero 2004) tienen un alto contenido de fibra que pueden ir de $1,4 \%$ a $8,3 \%$ dependiendo de la hortaliza a emplear que comparados con los resultados reportados en la presente investigación van desde $1,29 \%$ a $1,76 \%$ debido a que las hortalizas bulbos y tubérculos utilizados para la elaboración de la bebida tienen mayor contenido de agua que sólidos.

\section{Valoración Microbiológica}

Los resultados de los análisis microbiológicos y de acuerdo al ADEVA determinan que en los diferentes tratamientos hubo Ausencia de Coliformes Totales (NTE INEN 1529 -7) UCF/ ml, Ausencia de Escherichia Coli (NTE INEN 1529 -8) UCF/ ml y Ausencia de Salmonella (MÉTODO BETAS STAR) UCF/ $\mathrm{ml}$ de las bebidas analizadas, mientras que al tabular los análisis de Mohos y Levaduras (NTE INEN 1529-10) según el reporte se encontraron diferencias estadísticas. Conforme a los resultados obtenidos se deduce que no hubo contaminación debido a la asepsia con la que se realizó el producto mediante la implementación de un programa higiénico sanitario adecuado antes durante y después de la elaboración influyendo también el tratamiento térmico aplicado al producto que garantizó que las bebidas alarguen su tiempo de vida útil, la presencia de los mohos y levaduras encontrados en el producto fue mínima tomando la referencia (NTE INEN 1529-10) en la que se acepta la presencia de $100 \mathrm{UCF} / \mathrm{ml}$. de producto determinándose de acuerdo a los reportes que en ninguna muestra del producto en estudio se reportaron valores superiores a 8 $\mathrm{UCF} / \mathrm{gr}$.

Gráfico No. 2: Valoración microbiológica de Mohos y levaduras de la bebida fría nutritiva e hidratante elaborada con diferentes niveles de tomate y pepinillo.

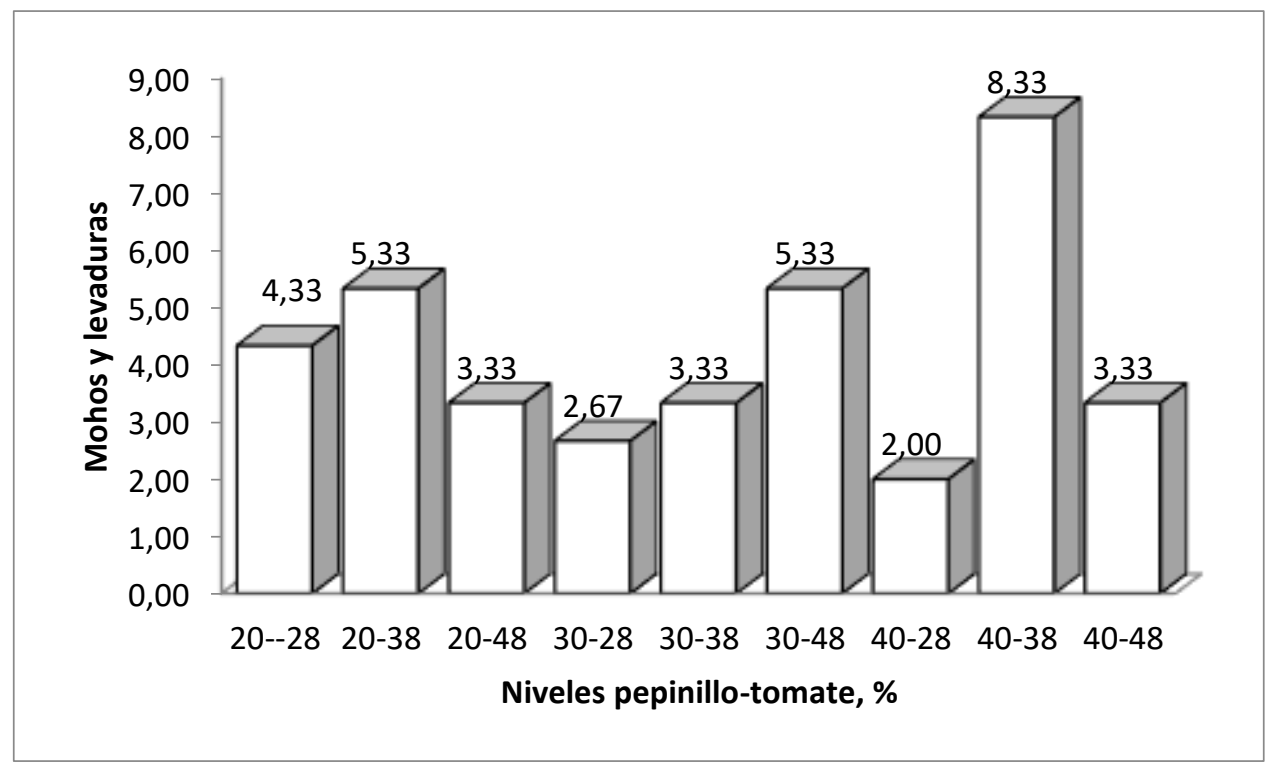

Fuente: Vallejo Sonia 2015 
Tabla N. 1: Valoración Microbiológica de la bebida fría nutritiva e hidratante elaborada con diferentes niveles de tomate y pepinillo.

\begin{tabular}{|c|c|c|c|c|c|c|}
\hline \multirow{2}{*}{$\begin{array}{l}\text { Niveles, \% } \\
\text { Pepinillo } \\
\mathrm{x}\end{array}$} & \multirow{2}{*}{ Tomate } & \multicolumn{5}{|c|}{ Parámetros, UFC/ml } \\
\hline & & $\begin{array}{l}\text { Coliformes } \\
\text { totales }\end{array}$ & $\begin{array}{l}\text { Escherichia } \\
\text { coli }\end{array}$ & \multicolumn{2}{|c|}{$\begin{array}{l}\text { Mohos y } \\
\text { levaduras }\end{array}$} & Salmonella \\
\hline 20 & 28 & Ausencia & Ausencia & 4,33 & $\mathrm{bc}$ & Ausencia \\
\hline 20 & 38 & Ausencia & Ausencia & 5,33 & $\mathrm{~b}$ & Ausencia \\
\hline 20 & 48 & Ausencia & Ausencia & 3,33 & $\mathrm{~cd}$ & Ausencia \\
\hline 30 & 28 & Ausencia & Ausencia & 2,67 & $\mathrm{~d}$ & Ausencia \\
\hline 30 & 38 & Ausencia & Ausencia & 3,33 & $\mathrm{~cd}$ & Ausencia \\
\hline 30 & 48 & Ausencia & Ausencia & 5,33 & $\mathrm{~b}$ & Ausencia \\
\hline 40 & 28 & Ausencia & Ausencia & 2,00 & d & Ausencia \\
\hline 40 & 38 & Ausencia & Ausencia & 8,33 & $\mathrm{a}$ & Ausencia \\
\hline 40 & 48 & Ausencia & Ausencia & 3,33 & $\mathrm{~cd}$ & Ausencia \\
\hline E.E. & & & & 0,497 & & \\
\hline Prob. & & & & 0,000 & & \\
\hline
\end{tabular}

Prob. $<0,01$ : Existen diferencias altamente significativas $(* *)$.

Medias con letras diferentes en la columna, difieren estadísticamente de acuerdo a la prueba de Duncan.

Fuente: Gráfico No. 1: Valoración microbiológica de Mohos y levaduras de la bebida fría nutritiva e hidratante elaborada con diferentes niveles de tomate y pepinillo.

\section{Evaluación Sensorial}

Para la evaluación sensorial de la bebida fría nutritiva e hidratante a base de hortalizas, bulbos y tubérculos se realizaron los respectivos análisis estadísticos y el ADEVA, basados en la siguiente escala numérica: Malo (0-4 puntos), Bueno (5-7 puntos), Muy Bueno (8-9 puntos) y Excelente (10 puntos).

El panel de catadores calificó la bebida bajo los siguientes parámetros predefinidos: Color (0-10 puntos), Olor (0-10 puntos), Sabor (0-10 puntos), Apariencia (0-10 puntos).

\section{Color}

Con respecto al color de la bebida fría e hidratante a base de hortalizas, bulbos y tubérculos que se calificó sobre 10 puntos, elaborada con pepinillo y diferentes niveles de tomate (28\%, $38 \%$ y $48 \%)$, se reportaron diferencias altamente significativas $(\mathrm{P}<0.01)$, por efecto de los niveles de tomate empleados obteniéndose el mayor puntaje 6,78 cuando se empleó $48 \%$ de tomate, sin embargo se observa también que existe un menor puntaje 5,00 cuando se utilizó en la formulación de la bebida el $38 \%$ de tomate, mientras que en la formulación de la bebida con tomate y diferentes niveles de pepinillo $(20 \%, 30 \%$ y $40 \%)$, se encontraron diferencias altamente significativas $(\mathrm{P}<0.01)$, por efecto de los niveles de pepinillo, obteniéndose un 
mayor puntaje 6,22 cuando se empleó $30 \%$ y un menor puntaje 5,33 cuando se utilizó en la formulación de la bebida el $20 \%$.

\section{Olor}

El olor de la bebida fría e hidratante a base de hortalizas, bulbos y tubérculos valorada sobre 10 puntos, elaborada con pepinillo y diferentes niveles de tomate $(28 \%$, $38 \%$ y $48 \%$ ), se reportó que no existen diferencias estadísticas (P.>0,05), por efecto de los niveles de tomate empleados obteniéndose el mayor puntaje 7,33 cuando se empleó 48 y 38\% de tomate, sin embargo se observa también que existe un menor puntaje 6,89 cuando se utilizó en la formulación de la bebida el $28 \%$ de tomate, mientras que en la formulación de la bebida con tomate y diferentes niveles de pepinillo $(20 \%, 30 \%$ y $40 \%)$, se reportó que no existen diferencias estadísticas (P.>0,05), por efecto de los niveles de pepinillo, obteniéndose un mayor puntaje 7,22 cuando se empleó 30y $40 \%$ de pepinillo y un menor puntaje 7,11 cuando se utilizó el $20 \%$ de pepinillo.

\section{Sabor}

El sabor de la bebida en estudio valorada sobre 10 puntos, elaborada con pepinillo y diferentes niveles de tomate $(28 \%, 38 \%$ y $48 \%)$, según los análisis estadísticos y de acuerdo al ADEVA, se reportaron diferencias significativas $(\mathrm{P}<0.01)$, por efecto de los niveles de tomate empleados obteniéndose el mayor puntaje 7,44 cuando se empleó $48 \%$ y un menor puntaje 6,33 cuando se utilizó en la formulación de la bebida el 38\%, mientras que en la formulación de la bebida con tomate y diferentes niveles de pepinillo (20\%, 30\% y 40\%), no se encontraron diferencias estadísticas (P.>0,05), por efecto de los niveles de pepinillo, obteniéndose un mayor puntaje 7 cuando se empleó $30 \%$ de pepinillo y un menor puntaje 6,77 cuando se utilizó en la formulación de la bebida el $40 \%$ de pepinillo.

\section{Apariencia}

En la evaluación de la apariencia sobre 10 puntos de la bebida fría e hidratante a base de hortalizas, bulbos y tubérculos elaborada con pepinillo y diferentes niveles de tomate (28\%, $38 \%$ y 48\%), según los análisis estadísticos y de acuerdo al ADEVA se reportaron diferencias altamente significativas $(\mathrm{P}<0.01)$, por efecto de los niveles de tomate utilizados obteniéndose el mayor puntaje 6 cuando se empleó $48 \%$ de tomate, observándose también que existe un menor puntaje 5 cuando se utilizó el 38\% de tomate, mientras que en la bebida con tomate y los niveles de pepinillo (20\%, 30\% y 40\%), empleados se encontraron diferencias altamente significativas $(\mathrm{P}<0.01)$, por efecto de los niveles de pepinillo lográndose obtener un mayor puntaje 5,67 cuando se empleó $40 \%$ y un menor puntaje 4,88 cuando se utilizó en la formulación de la bebida el $20 \%$ de pepinillo.

\section{Valoración Total}

En la valoración total sobre 40 puntos de la bebida elaborada con pepinillo y diferentes niveles de tomate, según los análisis estadísticos, se reportaron diferencias altamente 
significativas $(\mathrm{P}<0.01)$, por efecto de los niveles de tomate empleados $(28 \%, 38 \%$ y $48 \%)$ obteniéndose la mayor valoración 27,56 cuando se empleó $48 \%$ de tomate, sin embargo se observa también que existe una menor valoración 23,67 cuando se utilizó en la formulación de la bebida el $38 \%$ de tomate, mientras que en la formulación de la bebida con tomate y diferentes niveles de pepinillo (20\%, 30\% y 40\%), se encontraron diferencias significativas $(\mathrm{P}<0.01)$, por efecto de los niveles de pepinillo empleados obteniéndose una mayor valoración 26 cuando se empleó $30 \%$ de pepinillo y un menor puntaje 24,22 cuando se utilizó en la formulación de la bebida el $20 \%$ de pepinillo.

Por efecto de la interacción entre los niveles de pepinillo y tomate empleados se encontraron diferencias altamente significativas entre las medias determinadas, presentándose una mayor aceptación por la coloración de la bebida elaborada con $30 \%$ de pepinillo y $48 \%$ de tomate alcanzando una puntuación de 8 puntos sobre 10 , con similar comportamiento cuando se utilizaron los niveles 40 y $48 \%$ de pepinillo y tomate respectivamente, con una calificación de 7,67 puntos; por el contrario la bebida que menos aceptación tuvo fue la elaborada con el $20 \%$ de pepinillo y $48 \%$ de tomate recibiendo una valoración de apenas 4,67 puntos.

Tabla N. 2: Valoración Organoléptica del color, apariencia y total de la bebida fríanutritiva e hidratante elaborada con diferentes niveles de tomate y pepinillo.

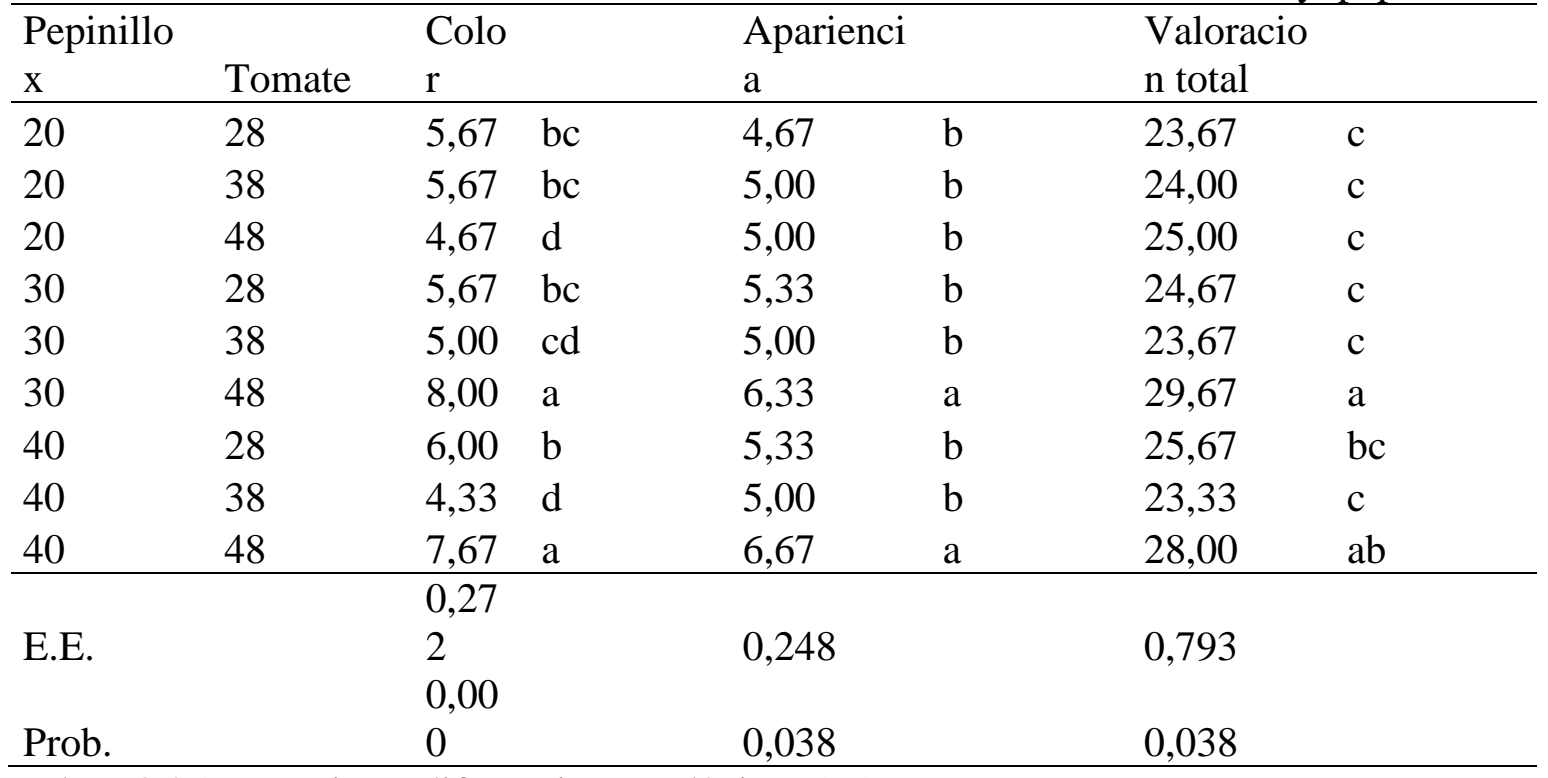

Prob. > 0,05: No existen diferencias estadísticas (ns).

Prob. $<0,01$ : Existen diferencias significativas $(*)$.

Prob. <0,01: Existen diferencias altamente significativas $(* *)$.

Medias con letras diferentes en la columna, difieren estadísticamente de acuerdo a la prueba de Duncan.

Fuente: Vallejo Sonia 2015 


\section{Conclusiones.}

- Por efecto de los niveles de pepinillo (20\%, 30\% y 40\%) se determinó que al utilizar el nivel con $40 \%$ de pepinillo en la bebida, el contenido de humedad aumenta $80,95 \%$ con respecto a los niveles 20 y $30 \%$, de la misma manera aumenta el contenido de proteína $2,74 \%$, grasa $15,37 \%$, fibra $1,6 \%$, cenizas $3.37 \%$ y pH 4.56 en este nivel y con respecto a los otros niveles, debido a que el pepinillo tiene un alto contenido de agua cerca del $96 \%$ y macronutrientes que hacen que la bebida sea altamente nutritiva.

- De la misma manera al evaluar sensorialmente las bebidas estudiadas por el efecto de los niveles de pepinillo se determinó que en la valoración total sobre 40 puntos la bebida con mayor aceptación fue aquella en la que se utilizó el 30\% de pepinillo obteniéndose un puntaje total de 26 puntos registrándose los valores más altos en comparación con los niveles $20(24,22)$ y $40 \%(25,66)$ respectivamente, de cada variable calificada sobre 10 tenemos que en coloración se obtuvo 6,22 puntos, 7,22 puntos de olor y 7 puntos de sabor a excepción de la apariencia en la que se obtuvo una puntuación de 5,55 más baja que la bebida con el 40\% de pepinillo 5,66 pero más alta que la bebida con el $20 \%$ de pepinillo 4,89 , debido a que la bebida a base de hortalizas es un producto nuevo la aceptación de la misma se ve reflejada en los resultados reportados en la que influyeron notablemente las variables de color, olor, sabor y apariencia del producto.

- Por efecto de los niveles de tomate $(28 \%, 38 \%$ y $48 \%)$ se determinó que al emplear el nivel $48 \%$ de tomate el contenido de humedad se incrementa a $81,12 \%$ al igual que el contenido de proteína $3,10 \%$, grasa $15,55 \%$, fibra 1,76 , cenizas $4,03 \%$ y $\mathrm{pH}$ 4,61 con respecto a los niveles 28 y $38 \%$ de tomate utilizados, obteniéndose resultados similares al factor analizado anteriormente debido a que el tomate también tiene un alto contenido de agua y nutrientes que aportaron a la bebida el valor nutricional esperado.

- En lo referente a la valoración organoléptica de la bebida en la que se evaluaron los diferentes niveles de tomate, se determinó que en la valoración total sobre 40 puntos la bebida mayormente aceptada fue la que alcanzó la puntuación de 27,56 puntos en la que se utilizó $48 \%$ de tomate cuyas variables valoradas sobre 10 puntos registraron los valores más altos con respecto a los niveles de tomate 28 y $38 \%$, debido a que el tomate proporciona mejores características sensoriales de color, olor, sabor y apariencia.

- En la valoración microbiológica y de acuerdo a los resultados de los análisis estadísticos para determinar la significancia de la presencia de los mohos y levaduras encontrados en el producto se tomó como referencia que es aceptable la presencia de $100 \mathrm{UCF} /$ gr. de producto, se determinó que en ninguna muestra del producto en estudio se reportaron valores superiores a $8 \mathrm{UCF} / \mathrm{gr}$ lo que significa que el tratamiento 
térmico aplicado al producto terminado se lo realizó correctamente mismo que garantizará que el producto tenga un largo período de vida útil.

\section{Referencias bibliográficas.}

al, Somogyi et. Tratamientos Térmicos aplicados a hortalizas y verduras. Torija, 1996.

AVI. «Food Analysis Laboratory Experiments.» En Food Analysis Laboratory

Experiments, de Ph.D. Clifton E. meloan, 79 -102. Manhattan, Kansas: Avi Publishing Company, inc., 1994.

Basque Molina, Elsa. Procesaalizasmieniento Térmico de frutas y hort. 2 da. Mexico: Trillas, 2012.

Buitrago, Ema Isabel Romero. «" Introducción de la zumoterapia en centros hospitalarios como complemento nutricional básico al tratamiento de patologías.".» Barranquilla, 2010, 16.

CAUDET YARZA, Francisco. Frutoterapia cura y prevención por medio de la fruta. Colección Cuadernos Alternativos, España: Editorial Astri S.A., 2001, 10.

Cedeño, Víctor Jesús Martín. «Consumo de hortalizas frescas en España.» Distribución y consumo, 2005: 5 .

De la Vega Ruiz, Gustavo. «Universidad Tecnológica de la Mixteca .» Mayo de 2009. http://www.utm.mx/edi_anteriores/Temas38/2NOTAS\%2038-1.pdf.

Dergal, Salvador Badui. Química de los alimentos. 4 ta. México: Grupo Herdez, S.A.

E, Falconí. «Plan De Recuperación Y Fomentos De Cultivos En Ecuador, Mediante El Desarrollo Y Produccción De Semillas Con Énfasis En Difusión De Variedades Mejoradas, Transferencia De Tenología Y Capacitación.»- Quito- Ecuador, 2008.

Fálder Rivero, Ángel. «Enciclopedia de los alimentos. Hortalizas (1).» Distribución y consumo, $\mathrm{n}^{\mathrm{o}} 75$ (2004): 129.

Felipe Durán Ramirez, María Fernanda Díaz Montes. Manual del Ingeniero en alimentos. Bogotá: Grupo Lstino Ltda, 2007.

Gaviola, Silvia. 4-18. «"Factores de manejo que inciden sobre la calidad de las hortalizas." .» Avances en Horticultura, 1996: 4 -18.

Gil, Angel. Tratado de Nutrición: Composición y Calidad Nutritiva de los Alimentos. Tomo II . Madrid: Medica Panamericana , 2010. 
Hernandez, Alicia. Microbiología Industrial . Madrid: EUNED, 2003.

Hernandez, Ángel Gil. «Tratado de Nutrición.» En Composición y Calidad nutritiva de los Alimentos, de Ángel Gil, 141-155. España: Medica panamericana, 2010.

INCAP. Industrias Nacionales de Salud. Tabla de Composición de Alimentos para uso en América LAtina. México, Argentina, Brasil, Colombia, Chile, Perú, Uruguay, Venezuela: Interamericana S.A.

INIAP. Poscosecha y Mercado de Chocho en Ecuador. Quito, 2001.

Instituto de Ciencias de la Salud, L.L.C. 819 N. Charles Street Baltimore, MD 21201. http://www.hsibaltimore.com. (último acceso: 19 de 04 de 2014).

Instituto de Investigaciones Agropecuarias "Jorge Dimitrov". Bayamo. Granma, Cuba. «ELABORACIÓN DE UNA BEBIDA FERMENTADA A PARTIR DEL SUERO.»Rev Cubana Aliment Nutr 2007;17(2):103-108, 2007.

Instituto Ecuatoriano de Normalilzación, INEN. «Norma Técnica Ecuatoriana. Sopas. Requisitos. NTE INEN 95:1979.» Documento Legal, Quito.

Jacoby, Enrique y Keller, Ingrid. «La promoción del consumo de frutas y verduras en América Latina: Buena oportunidad de acción intersectorial por una alimentación saludable.» Revista chilena de nutrición [online] 33 (2006): 226-231..

Lees, R. Análisis de los alimentos:. 3 ra. Zaragoza- España: Acribia.

Lopez, C. Alimentación y Nutrición . España: Diaz de Santos , 2005.

Marco R. Meyer, GaetanoPaltrinieri. Elaboración de frutas y hortalizas. México:

Trillas, 1999.

Martínez, Alfredo Gil. Preelaboración y conservación de los alimentos. Madrid: Akal. S.A, 2006.

Martínez, Iciar Astiasarán y J. Alfredo. Alimentos: Composición y Propieades. Editado por EDIGRAFOS. Madrid: MCGraw-Hill - Interamericana de España,S.A, 2000.

Ministerio de Agricultura. Gobierno de Chile .

http://www2.inia.cl/medios/biblioteca/ta/NR34251.pdf (último acceso: 10 de Septiembre de 2014).

MURCIA, José Luis, et al. , vol. 21, no 117, p. 63-103. «Alimentación en España: Hortalizas I.» Distribución y consumo 21, no 117 (2011): 63 - 103. 
Nidia Alba Cuéllar, Carlos Augusto Alba...et al. Ciencia, Tecnología e Industria de Alimentos. Bogotá: Grupo Latino Editores, 2008.

Perez, Jose María. Hostelería: Técnicas y Calidad de Servicio . Madrid: Eurocolor, 2011.

Potter, Norberto. La ciencia de los alimentos . México: Acribia, 1973.

Ronald S. Kirk, Ronald Sawyer, Harold egan. Composción y Análisis de Alimentos de Pearson. 2 da. México: Patria, 2008.

Salas , Jordi , Pilar García, y Jose Sanchez . La alimentación y la nutrición a traves de la Historia. Barcelona : Glosa, 2009.

Salgado, Nava, y Jimenez Munguía. Métodos de Control de Crecimiento Microbiano en el Pan . Puebla: Universidad de las Americas , 2012.

Sanchez, Ma. Teresa. Procesos de Elaboración de Alimentos y Bebidas . Madrid: Mundi Prensa, 2008.

Serrano, Ma. Antonia. «Accion de las acetogeninas de las anonaceas en las celulas cancerigenas.» Artículos de interés científico, 2009.

Torija. Valor nutritivo de las hortalizas y frutas. Zaragoza: Mc Gill, 2002.

Witting. Evaluación sensorial de alimentos. México: Trillas, 1991.

De la Vega Ruiz, Gustavo. «Universidad Tecnológica de la Mixteca .» Mayo de 2009. http://www.utm.mx/edi_anteriores/Temas38/2NOTAS\%2038-1.pdf.

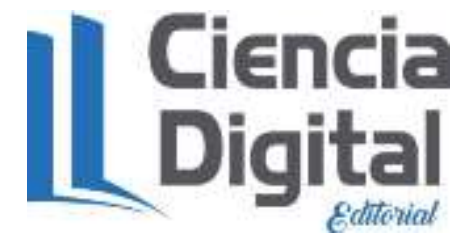




\section{PARA CITAR EL ARTÍCULO INDEXADO.}

Vallejo Abarca, S. M., \& Caluña Sánchez, E. (2019). Diseño de un proceso para la obtención de una bebida nutritiva a base de Hortalizas como el tomate (Solanum lycopersicum) y el pepinillo (Cucumis sativus). Ciencia Digital, 3(3), 464-480. https://doi.org/10.33262/cienciadigital.v3i3.662

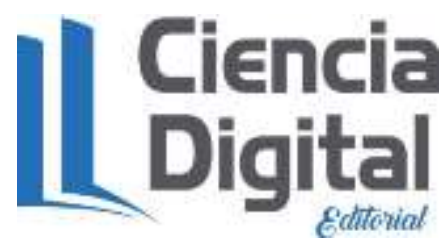

El artículo que se publica es de exclusiva responsabilidad de los autores y no necesariamente reflejan el pensamiento de la Revista Ciencia Digital.

El artículo queda en propiedad de la revista y, por tanto, su publicación parcial y/o total en otro medio tiene que ser autorizado por el director de la Revista Ciencia Digital.
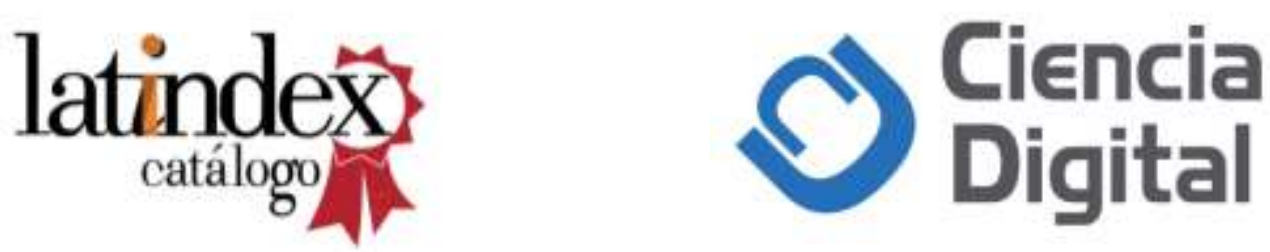